\title{
Sampled-Data Consensus for Nonlinear Multiagent Dynamical Systems via Reliable Control
}

\author{
Hongjie Li \\ College of Mathematics and Information and Engineering, Jiaxing University, Zhejiang 314001, China \\ Correspondence should be addressed to Hongjie Li; lhjymlly@163.com
}

Received 30 April 2014; Accepted 15 June 2014; Published 7 July 2014

Academic Editor: Chengjian Zhang

Copyright (C) 2014 Hongjie Li. This is an open access article distributed under the Creative Commons Attribution License, which permits unrestricted use, distribution, and reproduction in any medium, provided the original work is properly cited.

\begin{abstract}
The paper studies sampled-data consensus for nonlinear multiagent dynamical systems. A distributed linear reliable consensus protocol is designed, where probabilistic actuators with different failure rates and random network-induced delay are considered. Based on the input delay approach, a new distribution-based fault multiagent system model with random delay is proposed. By using the stochastic analysis technique and Kronecker product properties, some consensus conditions are derived in terms of linear matrix inequalities, and the solvability of derived conditions depends on not only the failure rate of the actuator but also on the probability of the delay. Finally, a numerical example is provided to demonstrate the effectiveness of the obtained theoretical results.
\end{abstract}

\section{Introduction}

In recent years, distributed coordination of multiagent systems has received increasing attention due to its potential applications, such as control of distributed sensor networks, unmanned-air-vehicle formations, satellite clusters, and so on [1-4]. As one of the fundamental and important issues in the area of cooperative control, the consensus problem in multiagent systems has been the hot and significant topic [5$13]$.

In many engineering applications, on the one hand, with the rapid development of computer hardware, only sampled data is used for the implementation of digital sensors, filters, and controllers. On the other hand, it is quite difficult to measure the continuous information transmission due to the capability of transmission bandwidth of networks; thus, it is more practical to apply sampled-data control, and the sampled-data control technology has shown more and more superiority over other control approaches. In particular, a new approach to deal with the sampled-data control problems has been proposed in [14-18]; the sampling period has been converted into a time-varying bounded delay. Based on this method, recently, many results have been established for the consensus in multiagent systems with sampled data. For example, in [19], the average consensus problem is considered for a class of first-order multiagent systems sampled-data communications; the consensus can be achieved by choosing the appropriate sampling interval. In $[11,20]$, some necessary and sufficient conditions are derived for consensus of multiagent systems with a fixed directed topology and periodic sampling. The consensus is also discussed for multiagent systems with sampled control by using zero-order holds or direction [21-24]. In [21], the sampled-data coordination algorithms are given for double-integrator dynamics, where the first algorithm guarantees that a team of vehicles achieves coordination on their positions with a zero final velocity and the second algorithm guarantees that a team of vehicles achieves coordination on their positions with a constant final velocity. The consensus problems for second-order agents in [22] are discussed in sampled-data setting, where the sampling period of each agent is independent of the others. Second-order sampled-data consensus problems are studied in $[23,24]$; the sampled position and velocity data are used in [23] to design the consensus protocol, but, in [24], only the sampled position data is utilized. It is well known that the agent needs to exchange the information with their neighbors by an interconnected network to accomplish some specific tasks. Hence, the wireless sensor networks play a critical role for information exchange, and in practice the sensor might be destroyed, subjected to external attacks, and fail to 
communicate with its neighbors; the reliability and robustness of multiagent systems might become weaker than before $[25,26]$. To describe the actuator fault, a Bernoulli distributed variable is employed to investigate the missing measurements $[27,28]$, which takes the value 0 to mean complete sensor failure and 1 to mean completely normal. Later, a more general random variable taking in $[0,1]$ is introduced in [2931 , when taking value in $(0,1)$ means the partial failure of the sensors. It should be pointed out that in most existing references concerning the multiagent systems, the related results have been obtained only by using the information of the variation range of the delay; however, in practice, the distribution of the delay is not uniform, the probability of the delay taking values in a long range may be very small [30-32]. This phenomenon motivates us to investigate the effect of the system performance. Unfortunately, up to now, by employing the distribution information of the time delay, designing the reliable consensus control algorithms by using the sampled data has not been investigated, and the purpose of this paper is to close this gap.

The main contributions of this paper can be summarized as follows. (1) A distributed linear reliable sampled-data consensus protocol is designed, where the actuator fault and random network-induced delay are considered. (2) A more generalized random variable is introduced for describing the different conditions of actuator fault; these conditions contain the case of complete failure, partial failure, complete normal, and measurements distortion. (3) The solvability of derived conditions depends on not only the size of the delay (the sampling period), but also on taking values of failure rates of the actuator fault.

Motivated by the above analysis, sampled-data consensus for nonlinear multiagent dynamical systems is investigated. A distributed linear reliable consensus protocol is designed, where probabilistic actuator and random delay are considered. Based on the input delay approach, some consensus conditions are derived in terms of linear matrix inequalities, containing the information of the actuator fault and random delay, the maximum sampling period (the delay) can be obtained. Finally, a numerical example is provided to demonstrate the effectiveness of the obtained theoretical results.

The rest of this paper is organized as follows. In Section 2, problem formulation and preliminaries are briefly outlined. In Section 3, main results are derived in the form of LMIs. In Section 4, a simulation example is provided to show the advantages of the obtained results, and some conclusions are drawn in Section 5.

Notation. The notation used in the paper is fairly standard. $\mathbb{R}^{n}$ denotes the $n$-dimensional Euclidean space and $\mathbb{R}^{n \times m}$ is a set of real $n \times m$ matrices. The notation $X>0$ (resp., $X<$ 0 ), for $X \in \mathbb{R}^{n \times n}$, means that the matrix $X$ is real symmetric positive definite (resp., negative definite). $\operatorname{diag}\{\cdots\}$ stands for a block-diagonal matrix. $\|\cdot\|$ denotes the Euclidean norm in $\mathbb{R}^{n}$. The superscript “ $T$ ” stands for matrix transposition. $I_{n}$ denotes $n \times n$ identity matrix. $E\{\cdot\}$ stands for mathematical expectation. The Kronecker product of matrices $Q \in \mathbb{R}^{m \times n}$ and $R \in \mathbb{R}^{p \times q}$ is a matrix in $\mathbb{R}^{m p \times n q}$ denoted as $Q \otimes R$. In this paper, if not explicitly stated, matrices are assumed to have compatible dimensions.

\section{Problem Formulation and Preliminaries}

Consider the following nonlinear multiagent dynamical systems consisting of $N$ agents

$$
\dot{x}(t)=A x_{i}(t)+f\left(x_{i}(t)\right)+u_{i}(t), \quad(i=1,2, \ldots, N),
$$

where $x_{i}(t) \in \mathbb{R}^{n}$ is the position state vector of the $i$ th agent. $f(\cdot) \in \mathbb{R}^{n}$ is an unknown but sector-bounded nonlinear vector function to describe the time-varying nonlinear dynamics of agent $i$ and $u_{i}(t) \in \mathbb{R}^{n}$ is the control input. In this paper, control input $u_{i}(t)$ is sampled before entering networks based on the sampling technique and zero-order hold circuit, and $t_{k}$ are the sampling instants satisfying $0 \leq t_{0}<t_{1}<\cdots<$ $t_{k}<\cdots$; for simplicity, it is assumed that $t_{k+1}-t_{k}=\tau$, where $\tau>0$ is the sampling period and then the controller can be described as

$$
\begin{aligned}
u_{i}(t) & =c K \sum_{j=1}^{N} a_{i j}\left[x_{j}\left(t_{k}\right)-x_{i}\left(t_{k}\right)\right] \\
& =-c K \sum_{j=1}^{N} l_{i j} x_{j}\left(t_{k}\right), \quad\left(t_{k} \leq t<t_{k+1}\right),
\end{aligned}
$$

where $\left[a_{i j}\right]_{N \times N}$ is a weighted adjacency matrix, $a_{i j}=0$ if and only if there is a connection between agent $i$ and agent $j$; otherwise $a_{i j}=0$. The Laplacian matrix $L=\left[l_{i j}\right]_{N \times N}$ is defined by $l_{i i}=-\sum_{j=1, j \neq i}^{N} l_{i j}$ and $l_{i j}=-a_{i j}(i \neq j) . c$ is a constant denoting the coupling strength; $K$ is the feedback controller to be designed.

Let $\tau(t)=t-t_{k}$. Then, (2) can be rewritten as

$$
u_{i}(t)=-c K \sum_{j=1}^{N} l_{i j} x_{j}(t-\tau(t)),
$$

where $0 \leq \tau(t)<\tau, \tau$ is the upper bound of the delay $\tau(t)$ which is also the sampling period.

When the actuator experiences failures, we use $u_{i}^{F}(t)$ to describe the control signal to be sent from actuator of the neighbors; that is,

$$
u_{i}^{F}(t)=-c \Pi K \sum_{j=1}^{N} l_{i j} x_{j}(t-\tau(t)),
$$

where $\Pi$ is a random variable taking values on the interval $[0, \theta]$, where $\theta \geq 1$. The mathematical expectation and variance of $\Pi$ are $\mu$ and $\sigma^{2}$, respectively.

Remark 1. Spring by [27-31], when $\Pi=0$, it means complete failure of actuator. When $\Pi=1$, it means the actuator has good working condition. When $0<\Pi<1$, it means partial failure of the actuator. When $\Pi>1$, it means the data distortion with the measurement of the actuator is bigger than the real measurement. 
Substituting (4) into (1), we can obtain

$$
\begin{gathered}
\dot{x}_{i}(t)=A x_{i}(t)+f\left(x_{i}(t)\right)-c \Pi K \sum_{j=1}^{N} l_{i j} x_{j}(t-\tau(t)) \\
=A x_{i}(t)+f\left(x_{i}(t)\right) \\
-c \mu K \sum_{j=1}^{N} l_{i j} x_{j}(t-\tau(t))-c(\Pi-\mu) K \\
\times \sum_{j=1}^{N} l_{i j} x_{j}(t-\tau(t)) .
\end{gathered}
$$

Assumption 2. The distribution of the random networkedinduced delay can be observed; that is, there exist constants $\tau_{1}$ and $\tau$, where $0 \leq \tau_{1} \leq \tau$, such that either $\tau(t) \in\left[0, \tau_{1}\right)$ or $\tau(t) \in\left[\tau_{1}, \tau\right]$, and the probability distribution of $\tau(t)$ taking values in $\left[0, \tau_{1}\right)$ and $\left[\tau_{1}, \tau\right]$ is known a priori.

Define the following two functions and a stochastic variable:

$$
\begin{gathered}
\tau_{1}(t)= \begin{cases}\tau(t), & \tau(t) \in\left[0, \tau_{1}\right), \\
\tau_{1}, & \tau(t) \in\left[\tau_{1}, \tau\right],\end{cases} \\
\tau_{2}(t)= \begin{cases}\tau(t), & \tau(t) \in\left[\tau_{1}, \tau\right], \\
\tau, & \tau(t) \in\left[0, \tau_{1}\right),\end{cases} \\
\alpha(t)= \begin{cases}1, & t \in\left\{t \mid \tau(t)=\tau_{1}(t)\right\}, \\
0, & t \in\left\{t \mid \tau(t)=\tau_{2}(t)\right\} .\end{cases}
\end{gathered}
$$

Assumption 3. $\alpha(t)$ is Bernoulli distributed sequence with $P\{\alpha(t)=1\}=\alpha_{0}$ and $P\{\alpha(t)=0\}=1-\alpha_{0}$. Then, we have $E\{\alpha(t)\}=\alpha_{0}$ and $E\left\{\left(\alpha(t)-\alpha_{0}\right)^{2}\right\}=\alpha_{0}\left(1-\alpha_{0}\right)$.

Remark 4. The Bernoulli distributed sequence is used to describe the distribution information of the delay in [33], which is an important characteristic of the network to affect the system performance. Unfortunately, such kind of statistical information has been utilized to design the consensus protocol for multiagent systems.

Combining (5)-(6), the system (6) can be rewritten as

$$
\begin{aligned}
& \dot{x}_{i}(t)= A x_{i}(t)+f\left(x_{i}(t)\right)-c \alpha(t) \mu K \\
& \times \sum_{j=1}^{N} l_{i j} x_{j}\left(t-\tau_{1}(t)\right)-c(1-\alpha(t)) \mu K \\
& \times \sum_{j=1}^{N} l_{i j} x_{j}\left(t-\tau_{2}(t)\right) \\
&-c \alpha(t)(\Pi-\mu) K
\end{aligned}
$$

$$
\begin{aligned}
& \times \sum_{j=1}^{N} l_{i j} x_{j}\left(t-\tau_{1}(t)\right)-c(1-\alpha(t))(\Pi-\mu) K \\
& \quad \times \sum_{j=1}^{N} l_{i j} x_{j}\left(t-\tau_{2}(t)\right) .
\end{aligned}
$$

By using the Kronecker product of the matrices, the above system (7) can be written in a compact form as

$$
\begin{aligned}
\dot{x}(t)= & \left(I_{N} \otimes A\right) x(t)+F(x(t))-c \mu \alpha(t)(L \otimes K) x \\
& \times\left(t-\tau_{1}(t)\right)-c \mu(1-\alpha(t))(L \otimes K) x\left(t-\tau_{2}(t)\right) \\
& -c \alpha(t)(\Pi-\mu)(L \otimes K) x\left(t-\tau_{1}(t)\right) \\
& -c(1-\alpha(t))(\Pi-\mu)(L \otimes K) x\left(t-\tau_{2}(t)\right)
\end{aligned}
$$

where

$$
\begin{gathered}
x(t)=\left(x_{1}^{T}(t), x_{2}^{T}(t), \ldots, x_{N}^{T}(t)\right)^{T}, \\
F(x(t))=\left(f^{T}\left(x_{1}(t)\right), f^{T}\left(x_{2}(t)\right), \ldots, f^{T}\left(x_{N}(t)\right)\right)^{T} .
\end{gathered}
$$

Before starting the main results, some definitions and lemmas are introduced here.

Definition 5. Consensus in multiagent systems (1) is said to be achieved if, for any initial conditions,

$$
\lim _{t \rightarrow \infty} E\left\|x_{i}(t)-x_{j}(t)\right\|=0, \quad(i, j=1,2, \ldots, N) .
$$

Assumption 6 (see [34]). For $\forall u, v \in \mathbb{R}^{n}$, the nonlinear function $f(\cdot)$ satisfies the following sector-bounded condition:

$$
\left[f(u)-f(v)-F_{1}(u-v)\right]^{T}\left[f(u)-f(v)-F_{1}(u-v)\right] \leq 0,
$$

where $F_{1}, F_{2}$ are real constant matrices with $F_{2}-F_{1} \geq 0$.

Lemma 7 (see $[35,36])$. Let $U=\left(u_{i j}\right)_{N \times N}, P \in \mathbb{R}^{n \times n}$, $x=\left(x_{1}^{T}, x_{2}^{T}, \ldots, x_{N}^{T}\right) \in \mathbb{R}^{N n}$, where $x_{i}=\left(x_{i 1}, x_{i 2}, \ldots, x_{i n}\right) \in$ $\mathbb{R}^{n}$ and $y=\left(y_{1}^{T}, y_{2}^{T}, \ldots, y_{N}^{T}\right) \in \mathbb{R}^{N n}$, where $y_{i}=$ $\left(y_{i 1}, y_{i 2}, \ldots, y_{i n}\right) \in \mathbb{R}^{n}$. If $U=U^{T}$ and each row sum of $U$ is zero, then

$$
x^{T}(U \otimes P) y=\sum_{1 \leq i<j \leq N} u_{i j}\left(x_{i}-x_{j}\right) P\left(y_{i}-y_{j}\right) .
$$

Lemma 8 (see [33]). $Q_{1 i}, Q_{2 i}(i=1,2)$ and $Q$ are some constant matrices of appropriate dimensions; $\tau_{i}(t)(i=1,2)$ satisfies $0 \leq \tau_{1}(t) \leq \tau_{1} \leq \tau_{2}(t) \leq \tau_{2}$. Then,

$$
\begin{aligned}
Q & +\left[\tau_{1}(t) Q_{11}+\left(\tau_{1}-\tau_{1}(t)\right) Q_{21}\right] \\
& +\left[\left(\tau_{2}(t)-\tau_{1}\right) Q_{12}+\left(\tau_{2}-\tau_{2}(t)\right) Q_{22}\right]<0
\end{aligned}
$$


if and only if

$$
\begin{aligned}
& Q+\tau_{1} Q_{11}+\left(\tau_{2}-\tau_{1}\right) Q_{12}<0, \\
& Q+\tau_{1} Q_{11}+\left(\tau_{2}-\tau_{1}\right) Q_{22}<0, \\
& Q+\tau_{1} Q_{21}+\left(\tau_{2}-\tau_{1}\right) Q_{12}<0, \\
& Q+\tau_{1} Q_{21}+\left(\tau_{2}-\tau_{1}\right) Q_{22}<0 .
\end{aligned}
$$

Lemma 9 (see $[35,36])$. Let $f(\cdot)$ be a nonnegative function defined on $[0,+\infty)$. If $f(\cdot)$ is Lebesgue integrable and is uniformly continuous on $[0,+\infty)$, then $\lim _{t \rightarrow \infty} f(t)=0$.

\section{Main Results}

In this section, we will deal with sampled-data consensus for nonlinear multiagent dynamical systems via reliable control.

Theorem 10. For given some positive scalars $\tau>\tau_{1}>0, \alpha_{0}>$ $0, \mu>0, c>0$ and the feedback gain matrix $K$, consensus in system (1) is achieved if there exist appropriate dimensional matrices $P>0, Q_{i}>0, R_{i}>0(i=1,2)$ and $Y_{i}, M_{i}, T_{i}$, $S_{i}(i=1,2)$, and a positive scalar $\alpha>0$, such that the following LMIs hold:

$$
\left[\begin{array}{cc}
\bar{\Sigma}_{11} & \bar{\Sigma}_{12}^{(l)} \\
* & \bar{\Sigma}_{22}
\end{array}\right]<0, \quad(1 \leq i<j \leq N ; l=1,2,3,4),
$$

where

$$
\begin{aligned}
& \bar{\Sigma}_{11}=\left[\begin{array}{cccccc}
\Sigma_{11} & \Sigma_{12} & \Sigma_{13} & 0 & \Sigma_{15} & 0 \\
* & \Sigma_{22} & 0 & 0 & 0 & 0 \\
* & * & \Sigma_{33} & \Sigma_{34} & 0 & 0 \\
* & * & * & \Sigma_{44} & \Sigma_{45} & 0 \\
* & * & * & * & \Sigma_{55} & \Sigma_{56} \\
* & * & * & * & * & \Sigma_{66}
\end{array}\right], \\
& \bar{\Sigma}_{12}^{(1)}=\left[\begin{array}{cccccc}
0 & 0 & \sqrt{\tau_{1}} M_{1}^{T} & \sqrt{\tau_{1}} M_{2}^{T} & 0 & 0 \\
0 & 0 & 0 & 0 & \sqrt{\left(\tau-\tau_{1}\right)} S_{1}^{T} & \sqrt{\left(\tau-\tau_{1}\right)} S_{2}^{T}
\end{array}\right]^{T}, \\
& \bar{\Sigma}_{12}^{(2)}=\left[\begin{array}{cccccc}
0 & 0 & \sqrt{\tau_{1}} M_{1}^{T} & \sqrt{\tau_{1}} M_{2}^{T} & 0 & 0 \\
0 & 0 & 0 & \sqrt{\left(\tau-\tau_{1}\right)} T_{1}^{T} & \sqrt{\left(\tau-\tau_{1}\right)} T_{2}^{T} & 0
\end{array}\right]^{T}, \\
& \bar{\Sigma}_{12}^{(3)}=\left[\begin{array}{cccccc}
\sqrt{\tau_{1}} Y_{1}^{T} & 0 & \sqrt{\tau_{1}} Y_{2}^{T} & 0 & 0 & 0 \\
0 & 0 & 0 & 0 & \sqrt{\left(\tau-\tau_{1}\right)} S_{1}^{T} & \sqrt{\left(\tau-\tau_{1}\right)} S_{2}^{T}
\end{array}\right]^{T}, \\
& \bar{\Sigma}_{12}^{(4)}=\left[\begin{array}{cccccc}
\sqrt{\tau_{1}} Y_{1}^{T} & 0 & \sqrt{\tau_{1}} Y_{2}^{T} & 0 & 0 \\
0 & 0 & 0 & \sqrt{\left(\tau-\tau_{1}\right)} T_{1}^{T} & \sqrt{\left(\tau-\tau_{1}\right)} T_{2}^{T} & 0
\end{array}\right]^{T}, \\
& \bar{\Sigma}_{22}=\operatorname{diag}\left\{-R_{1},-R_{2}\right\}, \\
& \Sigma_{11}=P A+A^{T} P+Q_{1}+Q_{2}+A^{T} R_{12} A \\
& +Y_{1}+Y_{1}^{T}-\alpha\left(F_{1}^{T} F_{2}+F_{2}^{T} F_{1}\right), \\
& \Sigma_{12}=P+A^{T} R_{12}+\alpha\left(F_{1}^{T}+F_{2}^{T}\right) \text {, } \\
& \Sigma_{13}=c \alpha_{0} \mu L_{i j} P K+c \alpha_{0} \mu N L_{i j} A^{T} R_{12} K-Y_{1}+Y_{2}^{T}, \\
& \Sigma_{15}=c\left(1-\alpha_{0}\right) \mu N L_{i j} P K+c\left(1-\alpha_{0}\right) \mu N L_{i j} A^{T} R_{12} K,
\end{aligned}
$$

$$
\begin{gathered}
\Sigma_{22}=R_{12}-2 \alpha I, \\
\Sigma_{33}=-2 c^{2} \alpha_{0}\left(\mu^{2}+\sigma^{2}\right) N L_{i j}^{(2)} K^{T} R_{12} K \\
-Y_{2}-Y_{2}^{T}+M_{1}+M_{1}^{T}, \\
\Sigma_{34}=-M_{1}+M_{2}^{T}, \\
\Sigma_{44}=-Q_{1}-M_{2}-M_{2}^{T}+T_{1}+T_{1}^{T}, \\
\Sigma_{45}=-T_{1}+T_{2}^{T}, \\
\Sigma_{55}=-2 c^{2}(1-\alpha)\left(\mu^{2}+\sigma^{2}\right) N L_{i j}^{(2)} K^{T} R_{12} K \\
-T_{2}-T_{2}^{T}+S_{1}+S_{1}^{T}, \\
\Sigma_{56}=-S_{1}+S_{2}^{T}, \\
\Sigma_{66}=-Q_{2}-S_{2}-S_{2}^{T}, \\
R_{12}=\tau_{1} R_{1}+\left(\tau-\tau_{1}\right) R_{2} .
\end{gathered}
$$

Proof. Construct a Lyapunov-Krasovskii functional candidate as

$$
V\left(t, x_{t}\right)=V_{1}\left(t, x_{t}\right)+V_{2}\left(t, x_{t}\right)+V_{3}\left(t, x_{t}\right),
$$

where

$$
\begin{gathered}
V_{1}\left(t, x_{t}\right)=x^{T}(t)(U \otimes P) x(t), \\
V_{2}\left(t, x_{t}\right)=\int_{t-\tau_{1}}^{t} x^{T}(s)\left(U \otimes Q_{1}\right) x(s) d s \\
\quad+\int_{t-\tau}^{t} x^{T}(s)\left(U \otimes Q_{2}\right) x(s) d s, \\
V_{3}\left(t, x_{t}\right)=\int_{t-\tau_{1}}^{t} \int_{s}^{t} \dot{x}^{T}(v)\left(U \otimes R_{1}\right) \dot{x}(v) d v d s \\
+\int_{t-\tau}^{t} \int_{s}^{t} \dot{x}^{T}(v)\left(U \otimes R_{3}\right) \dot{x}(v) d v d s,
\end{gathered}
$$

where $P, Q_{i}, R_{i}>0(i=1,2)$ are some matrices to be determined and

$$
U=\left[\begin{array}{ccccc}
N-1 & -1 & -1 & \cdots & -1 \\
-1 & N-1 & -1 & \cdots & -1 \\
-1 & -1 & N-1 & \cdots & -1 \\
\vdots & \vdots & \vdots & \ddots & \vdots \\
-1 & -1 & -1 & \cdots & N-1
\end{array}\right]
$$

The infinitesimal operator $\mathscr{L}$ of $V\left(t, x_{t}\right)$ is defined as

$$
\mathscr{L} V\left(t, x_{t}\right)=\lim _{\Delta \rightarrow 0^{+}} \frac{E\left\{\left\{V\left(t+\Delta, x_{t+\Delta}\right) \mid x_{t}\right\}-V\left(t, x_{t}\right)\right\}}{\Delta} .
$$


Calculating the derivative of $V_{i}\left(t, x_{t}\right)(i=1,2,3)$ along the trajectories of system (8), we have

$$
\begin{aligned}
\mathscr{L} V_{1}\left(t, x_{t}\right)= & 2 x^{T}(t)(U \otimes P) \\
& \times\left[\left(I_{N} \otimes A\right) x(t)+F(x(t))\right. \\
& -c \alpha_{0} \mu(L \otimes K) x\left(t-\tau_{1}(t)\right) \\
& \left.-c\left(1-\alpha_{0}\right) \mu(L \otimes K) x\left(t-\tau_{2}(t)\right)\right] \\
= & 2 x^{T}(t)(U \otimes P A) x(t) \\
& +2 x^{T}(t)(U \otimes P) F(x(t)) \\
& -2 c \alpha_{0} \mu x^{T}(t)(U L \otimes P K) x\left(t-\tau_{1}(t)\right) \\
& -2 c\left(1-\alpha_{0}\right) \mu x^{T}(t)(U L \otimes P K) x\left(t-\tau_{2}(t)\right), \\
\mathscr{L} V_{2}\left(t, x_{t}\right)= & x^{T}(t)\left[\left(U \otimes Q_{1}\right)+\left(U \otimes Q_{2}\right)\right] x(t) \\
& -x^{T}\left(t-\tau_{1}\right)\left(U \otimes Q_{1}\right) x\left(t-\tau_{1}\right) \\
& -x^{T}(t-\tau)\left(U \otimes Q_{2}\right) x(t-\tau), \\
& \mathscr{L} V_{3}\left(t, x_{t}\right) \\
& =\dot{x}^{T}(t)\left[\tau_{1}\left(U \otimes R_{1}\right)+\left(\tau-\tau_{1}\right)\right. \\
& -\int_{t-\tau}^{t-\tau_{1}} \dot{x}^{T}(s)\left(U \otimes R_{2}\right) \dot{x}(s) d s . \\
& \left.-\int_{t-\tau_{1}}^{t} \dot{x}^{T}(s)\left(U \otimes R_{2}\right)\right] \dot{x}(t)
\end{aligned}
$$

Denoting $R_{12}=\tau_{1} R_{1}+\left(\tau-\tau_{1}\right) R_{2}$, then $\tau_{1}\left(U \otimes R_{1}\right)+(\tau-$ $\left.\tau_{1}\right)\left(U \otimes R_{2}\right)=U \otimes R_{12}$, and recalling (8), we can obtain

$$
\begin{aligned}
E\left\{\dot{x}^{T}(t)(\right. & \left.\left.U \otimes R_{12}\right) \dot{x}(t)\right\} \\
=E & \left\{x^{T}(t)\left(I_{N} \otimes A\right)^{T}\left(U \otimes R_{12}\right)\left(I_{N} \otimes A\right) x(t)\right. \\
& +F^{T}(x(t))\left(U \otimes R_{12}\right) F(x(t)) \\
& +c^{2} \alpha_{0} \mu^{2} x^{T}\left(t-\tau_{1}(t)\right)(L \otimes K)^{T} \\
& \times\left(U \otimes R_{12}\right)(L \otimes K) x\left(t-\tau_{1}(t)\right) \\
& +c^{2}\left(1-\alpha_{0}\right) \mu^{2} x^{T}\left(t-\tau_{2}(t)\right)(L \otimes K)^{T} \\
& \times\left(U \otimes R_{12}\right)(L \otimes K) x\left(t-\tau_{2}(t)\right) \\
& +c^{2} \alpha_{0}(\pi-\mu)^{2} x^{T}\left(t-\tau_{1}(t)\right)(L \otimes K)^{T} \\
& \times\left(U \otimes R_{12}\right)(L \otimes K) x\left(t-\tau_{1}(t)\right) \\
& +c^{2}\left(1-\alpha_{0}\right)(\pi-\mu)^{2} x^{T}\left(t-\tau_{2}(t)\right)(L \otimes K)^{T} \\
& \times\left(U \otimes R_{12}\right)(L \otimes K) x\left(t-\tau_{2}(t)\right)
\end{aligned}
$$

$$
\begin{aligned}
& -2 x^{T}(t)\left(I_{N} \otimes A\right)^{T}\left(U \otimes R_{12}\right) \\
& \times\left[c \alpha_{0} \mu(L \otimes K) x\left(t-\tau_{1}(t)\right)\right. \\
& \left.\left.+c\left(1-\alpha_{0}\right) \mu(L \otimes K) x\left(t-\tau_{2}(t)\right)\right]\right\} \\
= & x^{T}(t)\left(U \otimes A^{T} R_{12} A\right) x(t) \\
+ & F^{T}(x(t))\left(U \otimes R_{12}\right) F(x(t)) \\
+ & c^{2} \alpha_{0} \mu^{2} x^{T}\left(t-\tau_{1}(t)\right) \\
\times & {\left[\left(L^{T} U L\right) \otimes\left(K^{T} R_{12} K\right)\right] x\left(t-\tau_{1}(t)\right) } \\
+ & c^{2} \alpha_{0} \sigma^{2} x^{T}\left(t-\tau_{1}(t)\right) \\
\times & {\left[\left(L^{T} U L\right) \otimes\left(K^{T} R_{12} K\right)\right] x\left(t-\tau_{1}(t)\right) } \\
+ & c^{2}\left(1-\alpha_{0}\right) \sigma^{2} x^{T}\left(t-\tau_{2}(t)\right) \\
\times & {\left[\left(L^{T} U L\right) \otimes\left(K^{T} R_{12} K\right)\right] x\left(t-\tau_{2}(t)\right) } \\
+ & 2 x^{T}(t)\left(U \otimes A^{T} R_{12}\right) F(x(t)) \\
- & 2 c \alpha_{0} \mu x^{T}(t)\left(U L \otimes A^{T} R_{12} K\right) x\left(t-\tau_{1}(t)\right) \\
- & 2 c\left(1-\alpha_{0}\right) \mu x^{T}(t)\left(U L \otimes A^{T} R_{12} K\right) x\left(t-\tau_{2}(t)\right) .
\end{aligned}
$$

Employing the free matrix method, we have

$$
\begin{gathered}
2 \xi^{T}(t)(U \otimes Y)\left[x(t)-x\left(t-\tau_{1}(t)\right)\right. \\
\left.-\int_{t-\tau_{1}(t)}^{t} \dot{x}(s) d s\right]=0, \\
2 \xi^{T}(t)(U \otimes M)\left[x\left(t-\tau_{1}(t)\right)-x\left(t-\tau_{1}\right)\right. \\
\left.-\int_{t-\tau_{1}}^{t-\tau_{1}(t)} \dot{x}(s) d s\right]=0, \\
2 \xi^{T}(t)(U \otimes T)\left[x\left(t-\tau_{1}\right)-x\left(t-\tau_{2}(t)\right)\right. \\
\left.-\int_{t-\tau_{2}(t)}^{t-\tau_{1}} \dot{x}(s) d s\right]=0, \\
{\left[x\left(t-\tau_{2}(t)\right)-x(t-\tau)\right.} \\
\left.-\int_{t-\tau}^{t-\tau_{2}(t)} \dot{x}(s) d s\right]=0
\end{gathered}
$$

where

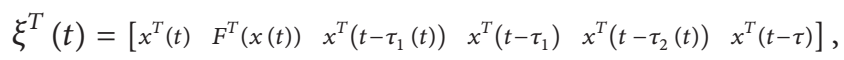

$$
\begin{aligned}
& Y^{T}=\left[\begin{array}{llllll}
Y_{1}^{T} & 0 & Y_{2}^{T} & 0 & 0 & 0
\end{array}\right],
\end{aligned}
$$




$$
\begin{aligned}
M^{T} & =\left[\begin{array}{llllll}
0 & 0 & M_{1}^{T} & M_{2}^{T} & 0 & 0
\end{array}\right], \\
T^{T} & =\left[\begin{array}{llllll}
0 & 0 & 0 & T_{1}^{T} & T_{2}^{T} & 0
\end{array}\right], \\
S^{T} & =\left[\begin{array}{llllll}
0 & 0 & 0 & 0 & S_{1}^{T} & S_{2}^{T}
\end{array}\right] .
\end{aligned}
$$

It can be shown from (23) that there exist $R_{i}>0(i=1,2)$, such that

$$
\begin{aligned}
& -2 \xi^{T}(t)(U \otimes Y) \int_{t-\tau_{1}(t)}^{t} \dot{x}(s) d s \\
& \leq \tau_{1}(t) \xi^{T}(t)(U \otimes Y)\left(U \otimes R_{1}\right)^{-1}(U \otimes Y)^{T} \xi(t) \\
& +\int_{t-\tau_{1}(t)}^{t} \dot{x}^{T}(s)\left(U \otimes R_{1}\right) \dot{x}(s) d s, \\
& -2 \xi^{T}(t)(U \otimes M) \int_{t-\tau_{1}}^{t-\tau_{1}(t)} \dot{x}(s) d s \\
& \leq\left(\tau_{1}-\tau_{1}(t)\right) \xi^{T}(t)(U \otimes M)\left(U \otimes R_{1}\right)^{-1} \\
& \times(U \otimes M)^{T} \xi(t)+\int_{t-\tau_{1}}^{t-\tau_{1}(t)} \dot{x}^{T}(s)\left(U \otimes R_{1}\right) \dot{x}(s) d s, \\
& -2 \xi^{T}(t)(U \otimes T) \int_{t-\tau_{2}(t)}^{t-\tau_{1}} \dot{x}(s) d s \\
& \leq\left(\tau_{2}(t)-\tau_{1}\right) \xi^{T}(t)(U \otimes T)\left(U \otimes R_{2}\right)^{-1} \\
& \times(U \otimes T)^{T} \xi(t)+\int_{t-\tau_{2}(t)}^{t-\tau_{1}} \dot{x}^{T}(s)\left(U \otimes R_{2}\right) \dot{x}(s) d s, \\
& -2 \xi^{T}(t)(U \otimes S) \int_{t-\tau}^{t-\tau_{2}(t)} \dot{x}(s) d s \\
& \leq\left(\tau-\tau_{2}(t)\right) \xi^{T}(t)(U \otimes S)\left(U \otimes R_{2}\right)^{-1}(U \otimes S)^{T} \xi(t) \\
& +\int_{t-\tau}^{t-\tau_{2}(t)} \dot{x}^{T}(s)\left(U \otimes R_{2}\right) \dot{x}(s) d s .
\end{aligned}
$$

For $\forall \alpha>0$, it can be derived from Assumption 6 that

$$
\begin{aligned}
& -\alpha\left[\begin{array}{c}
x(t) \\
F(x(t))
\end{array}\right]^{T} \\
& \quad \times\left[\begin{array}{cc}
U \otimes\left(F_{1}^{T} F_{2}+F_{2}^{T} F_{1}\right) & -U \otimes\left(F_{1}^{T}+F_{2}^{T}\right) \\
-U \otimes\left(F_{1}+F_{2}\right) & 2(U \otimes I)
\end{array}\right] \\
& \quad \times\left[\begin{array}{c}
x(t) \\
F(x(t))
\end{array}\right] \geq 0 .
\end{aligned}
$$

Noting $U L=L U=N L$, based on the properties of the Kronecker, we can obtain $L^{T} U L=N L^{T} L$. For presentation convenience, let $x_{i j}(t)=x_{i}(t)-x_{j}(t), f_{i j}(t)=f\left(x_{i}(t)\right)-$ $f\left(x_{j}(t)\right)$, and $\xi_{i j}(t)=\xi_{i}(t)-\xi_{j}(t)$. Adding (23) to the right of
(20) and substituting (26) and (27) into (20), using Lemma 7 , one has

$$
\mathscr{L} V\left(t, x_{t}\right)
$$$$
\leq \sum_{1 \leq i<j \leq N}\left\{2 x_{i j}^{T}(t) P A x_{i j}(t)\right.
$$$$
+2 x_{i j}^{T}(t) P f_{i j}(t)
$$$$
+2 c \alpha_{0} \mu N L_{i j} x_{i j}^{T} P K x_{i j}\left(t-\tau_{1}(t)\right)
$$$$
+2 c\left(1-\alpha_{0}\right) \mu N L_{i j} x_{i j}^{T} P K x_{i j}\left(t-\tau_{2}(t)\right)
$$$$
+x_{i j}^{T}(t)\left(Q_{1}+Q_{2}\right) x_{i j}(t)
$$$$
-x_{i j}^{T}\left(t-\tau_{1}\right) Q_{1} x_{i j}\left(t-\tau_{1}\right)
$$$$
-x_{i j}^{T}(t-\tau) Q_{2} x_{i j}(t-\tau)
$$$$
+x_{i j}^{T}(t) A^{T} R_{12} A x_{i j}(t)
$$$$
+f_{i j}^{T}(t) R_{12} f_{i j}(t)-2 c^{2} \alpha_{0} \mu^{2} N L_{i j}^{(2)} x_{i j}^{T}
$$$$
\times\left(t-\tau_{1}(t)\right) K^{T} R_{12} K x_{i j}\left(t-\tau_{1}(t)\right)
$$$$
-2 c^{2}\left(1-\alpha_{0}\right) \mu^{2} N L_{i j}^{(2)} x_{i j}^{T}\left(t-\tau_{2}(t)\right)
$$$$
\times K^{T} R_{12} K x_{i j}\left(t-\tau_{2}(t)\right)
$$$$
-2 c^{2} \alpha_{0} \sigma^{2} N L_{i j}^{(2)} x_{i j}^{T}\left(t-\tau_{1}(t)\right)
$$$$
\times K^{T} R_{12} K x_{i j}\left(t-\tau_{1}(t)\right)
$$$$
-2 c^{2}\left(1-\alpha_{0}\right) \sigma^{2} N L_{i j}^{(2)} x_{i j}^{T}\left(t-\tau_{2}(t)\right)
$$$$
\times K^{T} R_{12} K x_{i j}\left(t-\tau_{2}(t)\right)
$$$$
+2 x_{i j}^{T}(t) A^{T} R_{12} f_{i j}(t)+2 c \alpha_{0} \mu N L_{i j} x_{i j}^{T}(t)
$$$$
\times A^{T} R_{12} K x_{i j}\left(t-\tau_{1}(t)\right)
$$$$
+2 c\left(1-\alpha_{0}\right) \mu N L_{i j} x_{i j}^{T}(t) A^{T} R_{12} K x_{i j}\left(t-\tau_{2}(t)\right)
$$$$
+2 \xi_{i j}^{T}(t) Y\left(x_{i j}(t)-x_{i j}\left(t-\tau_{1}(t)\right)\right)
$$$$
+2 \xi_{i j}^{T}(t) M\left(x_{i j}\left(t-\tau_{1}(t)\right)-x_{i j}\left(t-\tau_{1}\right)\right)
$$$$
+2 \xi_{i j}^{T}(t) T\left(x_{i j}\left(t-\tau_{1}\right)-x_{i j}\left(t-\tau_{2}(t)\right)\right)
$$$$
+2 \xi_{i j}^{T}(t) S\left(x_{i j}\left(t-\tau_{2}(t)\right)-x_{i j}(t-\tau)\right)
$$$$
-\alpha\left[\begin{array}{c}
x_{i j}(t) \\
f_{i j}(t)
\end{array}\right]^{T}\left[\begin{array}{cc}
F_{1}^{T} F_{2}+F_{2}^{T} F_{1} & -\left(F_{1}^{T}+F_{2}^{T}\right) \\
-\left(F_{1}+F_{2}\right) & 2 I
\end{array}\right]
$$

$\times\left[\begin{array}{l}x_{i j}(t) \\ f_{i j}(t)\end{array}\right]+\tau_{1}(t) \xi_{i j}^{T}(t) Y R_{1}^{-1} Y^{T} \xi_{i j}(t)$ 


$$
\begin{aligned}
&+\left(\tau_{1}-\tau_{1}(t)\right) \xi_{i j}^{T}(t) M R_{1}^{-1} M^{T} \xi_{i j}(t) \\
&+\left(\tau_{2}(t)-\tau_{1}\right) \xi_{i j}^{T}(t) T R_{2}^{-1} T^{T} \xi_{i j}(t) \\
&\left.+\left(\tau-\tau_{2}(t)\right) \xi_{i j}^{T}(t) S R_{2}^{-1} S^{T} \xi_{i j}(t)\right\} \\
&=\sum_{1 \leq i<j \leq N} \xi_{i j}^{T}(t) \Xi \xi_{i j}(t)
\end{aligned}
$$

where $L_{i j}^{(2)}$ is the element of the $i$ th row and the $j$ th column of $L^{2}$.

By Schur complement and Lemma 8, we can obtain $\Xi<0$ and then it follows that

$$
\begin{aligned}
\mathscr{L} V\left(t, x_{t}\right) & \leq \lambda_{\max }(\Xi) \sum_{1 \leq i<j \leq N} E\left\{\left\|\xi_{i j}(t)\right\|^{2}\right\} \\
& \leq \lambda_{\max }(\Xi) \sum_{1 \leq i<j \leq N} E\left\{\left\|x_{i j}(t)\right\|^{2}\right\} .
\end{aligned}
$$

Therefore, we have

$$
E\{V(t)\}-E\{V(0)\} \leq \lambda_{\max }(\Xi) \int_{0}^{t} \sum_{1 \leq i<j \leq N} E\left\{\left\|x_{i j}(s)\right\|^{2}\right\} d s
$$

which implies that

$$
\int_{0}^{t} \sum_{1 \leq i<j \leq N} E\left\{\left\|x_{i j}(s)\right\|^{2}\right\} d s \leq-\frac{1}{\lambda_{\max }(\Xi)} E\{V(0)\}<+\infty .
$$

It is easy to see that $E\left\{\left\|x_{i j}(s)\right\|^{2}\right\}$ is uniformly continuous on $[0,+\infty)$, and, from Lemma 9 , one has

$$
\lim _{t \rightarrow+\infty} E\left\{\left\|x_{i}(t)-x_{j}(t)\right\|\right\}=0 .
$$

From Definition 5, it can be seen that consensus is achieved in system (1). The proof can be completed.

Remark 11. A sufficient condition is presented for consensus analysis of multiagent systems (1), which is an LMI condition when the gain $K$ is given. When the gain $K$ is a matrix variable to be determined, the above condition is not an LMI, we are now ready to consider the gain $K$ design problem. From Theorem 10, the following result can be derived easily.

Theorem 12. For given some positive scalars $\tau>\tau_{1}>0$, $\alpha_{0}>0, \mu>0, c>0, \varepsilon_{1}>0$, and $\varepsilon_{2}>0$, consensus in system (1) is achieved with feedback gain $K=P^{-1} V$. If there exist appropriate dimensional matrices $P>0, Q_{1}>0, Q_{2}>0$, $V, Y_{i}, M_{i}, T_{i}$, and $S_{i}(i=1,2)$, and a positive scalar $\alpha>0$, such that the following LMIs hold:

$$
\left[\begin{array}{cccc}
\Xi_{11} & \Xi_{12} & \Xi_{13} & \Xi_{14}^{(l)} \\
* & \Xi_{22} & 0 & 0 \\
* & * & \Xi_{33} & 0 \\
* & * & * & \Xi_{44}
\end{array}\right]<0, \quad(1 \leq i<j \leq N ; l=1,2,3,4),
$$

where

$$
\Xi_{11}=\left[\begin{array}{cccccc}
\theta_{11} & \theta_{12} & \theta_{13} & 0 & \theta_{15} & 0 \\
* & \theta_{22} & 0 & 0 & 0 & 0 \\
* & * & \theta_{33} & \theta_{34} & 0 & 0 \\
* & * & * & \theta_{44} & \theta_{45} & 0 \\
* & * & * & * & \theta_{55} & \theta_{56} \\
* & * & * & * & * & \theta_{66}
\end{array}\right]
$$

$\Xi_{12}=$

$$
\left[\begin{array}{cccc}
\sqrt{\alpha_{0} \tau_{1}} \varepsilon_{1} A^{T} P & \sqrt{\left(1-\alpha_{0}\right) \tau_{1}} \varepsilon_{1} A^{T} P & 0 & 0 \\
0 & 0 & 0 & 0 \\
\sqrt{\alpha_{0} \tau_{1} r_{2} \varepsilon_{1}} V^{T} & 0 & \sqrt{\alpha_{0} \tau_{1} r_{1}} \varepsilon_{1} V^{T} & 0 \\
0 & 0 & 0 \\
0 & \sqrt{\left(1-\alpha_{0}\right) \tau_{1} r_{2} \varepsilon_{1}} V^{T} & 0 & \sqrt{\left(1-\alpha_{0}\right) \tau_{1} r_{1} \varepsilon_{1}} V^{T} \\
0 & 0 & 0 & 0
\end{array}\right],
$$

$\Xi_{13}=$

$\left[\begin{array}{cccc}\sqrt{\alpha_{0}\left(\tau-\tau_{1}\right) \varepsilon_{2}} A^{T} P & \sqrt{\left(1-\alpha_{0}\right)\left(\tau-\tau_{1}\right)} \varepsilon_{2} A^{T} P & 0 & 0 \\ 0 & 0 & 0 \\ \sqrt{\alpha_{0}\left(\tau-\tau_{1}\right)} r_{2} \varepsilon_{2} V^{T} & 0 & 0 & 0 \\ 0 & 0 & \left.\sqrt{\alpha_{0}(\tau-\tau 1}\right) r_{1} \varepsilon_{2} V^{T} & 0 \\ 0 & \sqrt{\left(1-\alpha_{0}\right)\left(\tau-\tau_{1}\right) r_{2} \varepsilon_{2} V^{T}} & 0 & 0 \\ 0 & 0 & \sqrt{\left(1-\alpha_{0}\right)\left(\tau-\tau_{1}\right) r_{1} \varepsilon_{2} V^{T}}\end{array}\right]$,

$\Xi_{14}^{(1)}=\left[\begin{array}{cccccc}0 & 0 & \sqrt{\tau_{1}} M_{1}^{T} & \sqrt{\tau_{1}} M_{2}^{T} & 0 & 0 \\ 0 & 0 & 0 & 0 & \sqrt{\left(\tau-\tau_{1}\right)} S_{1}^{T} & \sqrt{\left(\tau-\tau_{1}\right)} S_{2}^{T}\end{array}\right]^{T}$,

$$
\Xi_{14}^{(2)}=\left[\begin{array}{cccccc}
0 & 0 & \sqrt{\tau_{1}} M_{1}^{T} & \sqrt{\tau_{1}} M_{2}^{T} & 0 & 0 \\
0 & 0 & 0 & \sqrt{\left(\tau-\tau_{1}\right)} T_{1}^{T} & \sqrt{\left(\tau-\tau_{1}\right)} T_{2}^{T} & 0
\end{array}\right]^{T},
$$$$
\Xi_{14}^{(3)}=\left[\begin{array}{cccccc}
\sqrt{\tau_{1}} Y_{1}^{T} & 0 & \sqrt{\tau_{1}} Y_{2}^{T} & 0 & 0 & 0 \\
0 & 0 & 0 & 0 & \sqrt{\left(\tau-\tau_{1}\right)} S_{1}^{T} & \sqrt{\left(\tau-\tau_{1}\right)} S_{2}^{T}
\end{array}\right]^{T},
$$$$
\Xi_{14}^{(4)}=\left[\begin{array}{cccccc}
\sqrt{\tau_{1}} Y_{1}^{T} & 0 & \sqrt{\tau_{1}} Y_{2}^{T} & 0 & 0 & 0 \\
0 & 0 & 0 & \sqrt{\left(\tau-\tau_{1}\right)} T_{1}^{T} & \sqrt{\left(\tau-\tau_{1}\right)} T_{2}^{T} & 0
\end{array}\right]^{T},
$$

$$
\Xi_{22}=\operatorname{diag}\left\{-\varepsilon_{1} P,-\varepsilon_{1} P,-\varepsilon_{1} P,-\varepsilon_{1} P\right\},
$$$$
\Xi_{33}=\operatorname{diag}\left\{-\varepsilon_{2} P,-\varepsilon_{2} P,-\varepsilon_{2} P,-\varepsilon_{2} P\right\} \text {, }
$$$$
\Xi_{44}=\operatorname{diag}\left\{-\varepsilon_{1} P,-\varepsilon_{2} P\right\},
$$$$
\theta_{11}=P A+A^{T} P+Q_{1}+Q_{2}+Y_{1}
$$$$
+Y_{1}^{T}-\alpha\left(F_{1}^{T} F_{2}+F_{2}^{T} F_{1}\right),
$$$$
\theta_{12}=P+\tau_{1} \varepsilon_{1} A^{T} P+\left(\tau-\tau_{1}\right) \varepsilon_{2} A^{T} P
$$$$
+\alpha\left(F_{1}^{T}+F_{2}^{T}\right),
$$$$
\theta_{13}=\alpha_{0} r_{2} V-Y_{1}+Y_{2}^{T} \text {, }
$$$$
\theta_{15}=\left(1-\alpha_{0}\right) r_{2} V \text {, }
$$$$
\theta_{22}=\tau_{1} \varepsilon_{1} P+\left(\tau-\tau_{1}\right) \varepsilon_{2} P-2 \alpha I,
$$

$$
\begin{gathered}
\theta_{33}=-Y_{2}-Y_{2}^{T}+M_{1}+M_{1}^{T}, \\
\theta_{34}=-M_{1}+M_{2}^{T}, \\
\theta_{44}=-Q_{1}-M_{2}-M_{2}^{T}+T_{1}+T_{1}^{T},
\end{gathered}
$$




$$
\begin{gathered}
\theta_{45}=-T_{1}+T_{2}^{T}, \\
\theta_{55}=-T_{2}-T_{2}^{T}+S_{1}+S_{1}^{T}, \\
\theta_{56}=-S_{1}+S_{2}^{T}, \\
\theta_{66}=-Q_{2}-S_{2}-S_{2}^{T}, \\
r_{1}=-c^{2} \mu^{2} N^{2} L_{i j}^{2}-2 c^{2} \mu^{2} N L_{i j}^{(2)}-2 c^{2} \sigma^{2} N L_{i j}^{(2)}, \\
r_{2}=c \mu N L_{i j},
\end{gathered}
$$

then we can obtain the gain matrix $K=P^{-1} V$.

Proof. From (22) and Lemma 7, the following inequalities hold:

$$
\begin{aligned}
& E\left\{\dot{x}^{T}(t)\left(U \otimes R_{12}\right) \dot{x}(t)\right\} \\
& =\sum_{1 \leq i<j \leq N}\left\{x_{i j}^{T}(t) A^{T} R_{12} A x_{i j}(t)\right. \\
& -2 c^{2} \alpha_{0} \mu^{2} N L_{i j}^{(2)} x_{i j}^{T}\left(t-\tau_{1}(t)\right) \\
& \times K^{T} R_{12} K x_{i j}\left(t-\tau_{1}(t)\right) \\
& +f_{i j}^{T}(t) R_{12} f_{i j}(t)-2 c^{2}\left(1-\alpha_{0}\right) \mu^{2} N L_{i j}^{(2)} x_{i j}^{T} \\
& \times\left(t-\tau_{2}(t)\right) K^{T} R_{12} K x_{i j}\left(t-\tau_{2}(t)\right) \\
& -2 c^{2} \alpha_{0} \sigma^{2} N L_{i j}^{(2)} x_{i j}^{T}\left(t-\tau_{1}(t)\right) \\
& \times K^{T} R_{12} K x_{i j}\left(t-\tau_{1}(t)\right) \\
& -2 c^{2}\left(1-\alpha_{0}\right) \sigma^{2} N L_{i j}^{(2)} x_{i j}^{T}\left(t-\tau_{2}(t)\right) \\
& \times K^{T} R_{12} K x_{i j}\left(t-\tau_{2}(t)\right) \\
& +2 x_{i j}^{T}(t) A^{T} R_{12} f_{i j}(t)+2 c \alpha_{0} \mu N L_{i j} x_{i j}^{T}(t) \\
& \times A^{T} R_{12} K x_{i j}\left(t-\tau_{1}(t)\right)+2 c\left(1-\alpha_{0}\right) \\
& \left.\times \mu N L_{i j} x_{i j}^{T}(t) A^{T} R_{12} K x_{i j}\left(t-\tau_{2}(t)\right)\right\} \\
& =\sum_{1 \leq i<j \leq N}\left\{f_{i j}^{T}(t) R_{12} f_{i j}(t)+2 x_{i j}^{T}(t) A^{T} R_{12} f_{i j}(t)\right. \\
& +\alpha_{0} x_{i j}^{T}(t) A^{T} R_{12} A x_{i j}(t) \\
& +2 c \alpha_{0} \mu N L_{i j} x_{i j}^{T}(t) A^{T} R_{12} K x_{i j}\left(t-\tau_{1}(t)\right) \\
& +\alpha_{0} c^{2} \mu^{2} N^{2} L_{i j} x_{i j}^{T}\left(t-\tau_{1}(t)\right) \\
& \times K^{T} R_{12} K x_{i j}\left(t-\tau_{1}(t)\right) \\
& +\left(1-\alpha_{0}\right) x_{i j}^{T}(t) A^{T} R_{12} A x_{i j}(t)
\end{aligned}
$$

$$
\begin{aligned}
+ & 2 c\left(1-\alpha_{0}\right) \mu N L_{i j} x_{i j}^{T}(t) A^{T} R_{12} K x_{i j}\left(t-\tau_{2}(t)\right) \\
+ & \left(1-\alpha_{0}\right) c^{2} \mu^{2} N^{2} L_{i j}^{2} x_{i j}^{T}\left(t-\tau_{2}(t)\right) \\
\times & K^{T} R_{12} K x_{i j}\left(t-\tau_{2}(t)\right) \\
& -\left[\alpha_{0} c^{2} \mu^{2} N^{2} L_{i j}^{2}+2 \alpha_{0} c^{2} \mu^{2} N L_{i j}^{(2)}\right. \\
& \left.+2 \alpha_{0} c^{2} \sigma^{2} N L_{i j}^{(2)}\right] x_{i j}^{T} \\
& \times\left(t-\tau_{1}(t)\right) K^{T} R_{12} K x_{i j}\left(t-\tau_{1}(t)\right) \\
& -\left[\left(1-\alpha_{0}\right) c^{2} \mu^{2} N^{2} L_{i j}^{2}+2\left(1-\alpha_{0}\right) c^{2} \mu^{2} N L_{i j}^{(2)}\right. \\
& \left.+2\left(1-\alpha_{0}\right) c^{2} \sigma^{2} N L_{i j}^{(2)}\right] x_{i j}^{T} \\
& \left.\times\left(t-\tau_{2}(t)\right) K^{T} R_{12} K x_{i j}\left(t-\tau_{2}(t)\right)\right\} \\
\sum_{1 \leq i<j \leq N} & f_{i j}^{T}(t) R_{12} f_{i j}(t)+2 x_{i j}^{T}(t) A^{T} R_{12} f_{i j}(t) \\
& +\alpha_{0} \xi_{i j}^{T}(t) \mathscr{A}_{1}^{T} R_{12} \mathscr{A}_{1} \xi_{i j}(t) \\
+ & \left(1-\alpha_{0}\right) \xi_{i j}^{T}(t) \mathscr{A}_{2}^{T} R_{12} \mathscr{A}_{2} \xi_{i j}(t) \\
+ & \alpha_{0} r_{1} \xi_{i j}^{T}(t) \mathscr{A}_{3}^{T} R_{12} \mathscr{A}_{3} \xi_{i j}(t) \\
+ & \left.\left(1-\alpha_{0}\right) r_{1} \xi_{i j}^{T}(t) \mathscr{A}_{4}^{T} R_{12} \mathscr{A}_{4} \xi_{i j}(t)\right\} \\
& \\
+ &
\end{aligned}
$$

where

$$
\begin{gathered}
\mathscr{A}_{1}=\left[\begin{array}{llllll}
A & 0 & r_{2} K & 0 & 0 & 0
\end{array}\right]^{T}, \\
\mathscr{A}_{2}=\left[\begin{array}{llllll}
A & 0 & 0 & 0 & r_{2} K & 0
\end{array}\right]^{T}, \\
\mathscr{A}_{3}=\left[\begin{array}{llllll}
0 & 0 & K & 0 & 0 & 0
\end{array}\right]^{T}, \\
\mathscr{A}_{4}=\left[\begin{array}{llllll}
0 & 0 & 0 & 0 & K & 0
\end{array}\right]^{T}, \\
r_{1}=-c^{2} \mu^{2} N^{2} L_{i j}^{2}-2 c^{2} \mu^{2} N L_{i j}^{(2)}-2 c^{2} \sigma^{2} N L_{i j}^{(2)}, \\
r_{2}=c \mu N L_{i j} .
\end{gathered}
$$

Combining (28) and (35), we can obtain

$$
\begin{aligned}
& \mathscr{L} V\left(t, x_{t}\right) \\
& \leq \sum_{1 \leq i<j \leq N}\left\{2 x_{i j}^{T}(t) P A x_{i j}(t)+2 x_{i j}^{T}(t) P f_{i j}(t)\right. \\
& +2 c \alpha_{0} \mu N L_{i j} x_{i j}^{T} P K x_{i j}\left(t-\tau_{1}(t)\right) \\
& +2 c\left(1-\alpha_{0}\right) \mu N L_{i j} x_{i j}^{T} P K x_{i j}\left(t-\tau_{2}(t)\right) \\
& +x_{i j}^{T}(t)\left(Q_{1}+Q_{2}\right) x_{i j}(t) \\
& -x_{i j}^{T}\left(t-\tau_{1}\right) Q_{1} x_{i j}\left(t-\tau_{1}\right)-x_{i j}^{T}(t-\tau) \\
& \times Q_{2} x_{i j}(t-\tau)+x_{i j}^{T}(t) A^{T} R_{12} A x_{i j}(t)
\end{aligned}
$$




$$
\begin{aligned}
& +f_{i j}^{T}(t) R_{12} f_{i j}(t)+2 x_{i j}^{T}(t) A^{T} R_{12} f_{i j}(t) \\
& +\alpha_{0} \xi_{i j}^{T}(t) \mathscr{A}_{1}^{T} R_{12} \mathscr{A}_{1} \xi_{i j}(t) \\
& +\left(1-\alpha_{0}\right) \xi_{i j}^{T}(t) \mathscr{A}_{2}^{T} R_{12} \mathscr{A}_{2} \xi_{i j}(t) \\
& +\alpha_{0} r_{1} \xi_{i j}^{T}(t) \mathscr{A}_{3}^{T} R_{12} \mathscr{A}_{3} \xi_{i j}(t) \\
& +\left(1-\alpha_{0}\right) r_{1} \xi_{i j}^{T}(t) \mathscr{A}_{4}^{T} R_{12} \mathscr{A}_{4} \xi_{i j}(t) \\
& +2 \xi_{i j}^{T}(t) Y\left(x_{i j}(t)-x_{i j}\left(t-\tau_{1}(t)\right)\right) \\
& +2 \xi_{i j}^{T}(t) M\left(x_{i j}\left(t-\tau_{1}(t)\right)-x_{i j}\left(t-\tau_{1}\right)\right) \\
& +2 \xi_{i j}^{T}(t) T\left(x_{i j}\left(t-\tau_{1}\right)-x_{i j}\left(t-\tau_{2}(t)\right)\right) \\
& +2 \xi_{i j}^{T}(t) S\left(x_{i j}\left(t-\tau_{2}(t)\right)-x_{i j}(t-\tau)\right) \\
& -\alpha\left[\begin{array}{c}
x_{i j}(t) \\
f_{i j}(t)
\end{array}\right]^{T} \\
& \times\left[\begin{array}{cc}
F_{1}^{T} F_{2}+F_{2}^{T} F_{1} & -\left(F_{1}^{T}+F_{2}^{T}\right) \\
-\left(F_{1}+F_{2}\right) & 2 I
\end{array}\right] \\
& \times\left[\begin{array}{l}
x_{i j}(t) \\
f_{i j}(t)
\end{array}\right] \\
& +\tau_{1}(t) \xi_{i j}^{T}(t) Y R_{1}^{-1} Y^{T} \xi_{i j}(t)+\left(\tau_{1}-\tau_{1}(t)\right) \\
& \times \xi_{i j}^{T}(t) M R_{1}^{-1} M^{T} \xi_{i j}(t) \\
& +\left(\tau_{2}(t)-\tau_{1}\right) \xi_{i j}^{T}(t) T R_{2}^{-1} T^{T} \xi_{i j}(t) \\
& \left.+\left(\tau-\tau_{2}(t)\right) \xi_{i j}^{T}(t) S R_{2}^{-1} S^{T} \xi_{i j}(t)\right\} \\
& =\sum_{1 \leq i<j \leq N} \xi_{i j}^{T}(t) \bar{\Xi} \xi_{i j}(t) .
\end{aligned}
$$

Let $R_{1}=\varepsilon_{1} P, R_{2}=\varepsilon_{2} P$, and $V=P K$ in (15) and then by using Lemma 8 , we can obtain $\bar{\Xi}<0$. The proof is completed.

Remark 13. The solvability of derived conditions depends on not only the failure rate of the actuator but also on the probability of the delay. Since more information has been employed, less conservative results might be obtained, which can be seen from the following numerical example.

\section{Numerical Examples}

Example 14. For simplicity, we consider multiagent systems with three agents and the state vector of each node being
TABLE 1: The upper bounds of the delay (sampling period) for different values of $\alpha_{0}$.

\begin{tabular}{ccccccc}
\hline$\alpha_{0}$ & 0.1 & 0.3 & 0.5 & 0.7 & 0.9 & 0.99 \\
\hline$\tau$ & 0.0129 & 0.0204 & 0.0372 & 0.1063 & 0.3853 & 0.7250 \\
\hline
\end{tabular}

two dimensional; that is, $N=3, n=2$, and other related parameters are given as follows:

$$
\begin{gathered}
A=\left[\begin{array}{ll}
-4 & 1 \\
-2 & 4
\end{array}\right], \quad L=\left[\begin{array}{ccc}
2 & -1 & -1 \\
-1 & 2 & -1 \\
-1 & -1 & 2
\end{array}\right], \quad c=0.1, \\
\mu=1.4, \quad \sigma=0.2, \\
\tau_{1}=0.001, \quad \varepsilon_{1}=\varepsilon_{2}=0.1 .
\end{gathered}
$$

The nonlinear function is as follows:

$$
f\left(x_{i}(t)\right)=\left[\begin{array}{c}
0.5 x_{i 1}(t)-\tanh \left(0.2 x_{i 1}(t)\right)+0.2 x_{i 2}(t) \\
0.95 x_{i 2}(t)-\tanh \left(0.75 x_{i 2}(t)\right)
\end{array}\right]
$$

and then it is easy to verify that

$$
F_{1}=\left[\begin{array}{cc}
0.3 & 0.2 \\
0 & 0.2
\end{array}\right], \quad F_{2}=\left[\begin{array}{cc}
0.5 & 0.2 \\
0 & 0.95
\end{array}\right] .
$$

When the delay is random and its probability distribution is known, we can obtain the upper bound of the delay (sampling period) by Theorem 10; it is found in Table 1. It can be seen that when the probability information of the delay values in interval is known, the obtained upper bound of the delay (sampling period) is larger than the one obtained by tradition method with only variation range information $(\tau=0.0106)$ of the delay.

To illustrate the efficiency of the designed method, the following three possible cases are considered.

Case 1. Consider no actuator failure and measurements distortion in the system, that is $\mu=1, \sigma=0$, when $\alpha_{0}=0.7$. The upper bound of the delay (sampling period) is $\tau=0.1071$ by using Theorem 12, and the corresponding feedback gain is

$$
K=\left[\begin{array}{cc}
35.9117 & 21.0898 \\
-161.3924 & -243.4319
\end{array}\right] \text {. }
$$

The state responses are shown in Figure 1.

Case 2. When the actuator is partial failure, let $\mu=0.7, \sigma=$ 0.2 , and when $\alpha_{0}=0.7$, we can obtain that the upper bound of the delay (sampling period) is $\tau=0.1060$ and the feedback gain is

$$
K=\left[\begin{array}{cc}
50.4724 & 29.7689 \\
-224.4166 & -343.5873
\end{array}\right]
$$

The state responses are shown in Figure 2.

Case 3. When considering actuator fault and measurement distortion, let $\mu=1.4, \sigma=0.2$, and when $\alpha_{0}=0.7$, from 
TABLE 2: The upper bounds of the delay (sampling period) for different values of $\alpha_{0}$ and the conditions of actuator.

\begin{tabular}{lccccrr}
\hline$\alpha_{0}$ & 0.5 & 0.6 & 0.7 & 0.8 & 0.9 & 0.99 \\
\hline$\mu=1, \sigma=0$ & 0.0373 & 0.0569 & 0.1071 & 0.1988 & 0.3906 & 0.7457 \\
$\mu=0.7, \sigma=0.2$ & 0.0371 & 0.0560 & 0.1060 & 0.1915 & 0.3702 & 0.6691 \\
$\mu=1.4, \sigma=0.2$ & 0.0372 & 0.0567 & 0.1063 & 0.1969 & 0.3853 & 0.7250 \\
\hline
\end{tabular}

TABLE 3: The feedback gain matrices $K$ for different values of $\alpha_{0}$ and the conditions of actuator.

\begin{tabular}{|c|c|c|c|c|}
\hline$\alpha_{0}$ & 0.5 & 0.6 & 0.7 & 0.8 \\
\hline $\begin{array}{l}\mu=1 \\
\sigma=0\end{array}$ & {$\left[\begin{array}{cc}14.4864 & 9.5364 \\
-35.6942 & -108.7951\end{array}\right]$} & {$\left[\begin{array}{cc}22.8536 & 14.2459 \\
-73.8533 & -163.0010\end{array}\right]$} & {$\left[\begin{array}{cc}35.9117 & 21.0898 \\
-161.3924 & -243.4319\end{array}\right.$} & {$\left[\begin{array}{cc}62.6971 & 36.2678 \\
-419.9275 & -428.9063\end{array}\right.$} \\
\hline $\begin{aligned} \mu & =0.7 \\
\sigma & =0.2\end{aligned}$ & {$\left[\begin{array}{cc}21.0127 & 13.6887 \\
-51.8329 & -155.9968\end{array}\right.$} & {$\left[\begin{array}{cc}32.0593 & 20.0371 \\
-102.4567 & -229.2493\end{array}\right.$} & {$\left[\begin{array}{cc}50.4724 & 29.7689 \\
-224.4166 & -343.5873\end{array}\right.$} & {$\left[\begin{array}{cc}90.1993 & 53.0314 \\
-609.1302 & -628.2796\end{array}\right.$} \\
\hline $\begin{array}{l}\mu=1.4 \\
\sigma=0.2\end{array}$ & {$\left[\begin{array}{cc}10.2856 & 6.7829 \\
-25.2842 & -77.3933\end{array}\right]$} & {$\left[\begin{array}{cc}16.2402 & 10.1321 \\
-52.3181 & -115.9304\end{array}\right]$} & {$\left[\begin{array}{cc}25.5233 & 14.9789 \\
-114.2535 & -172.8522\end{array}\right.$} & {$\left[\begin{array}{cc}44.7015 & 25.9764 \\
-229.7514 & -307.3281\end{array}\right.$} \\
\hline
\end{tabular}

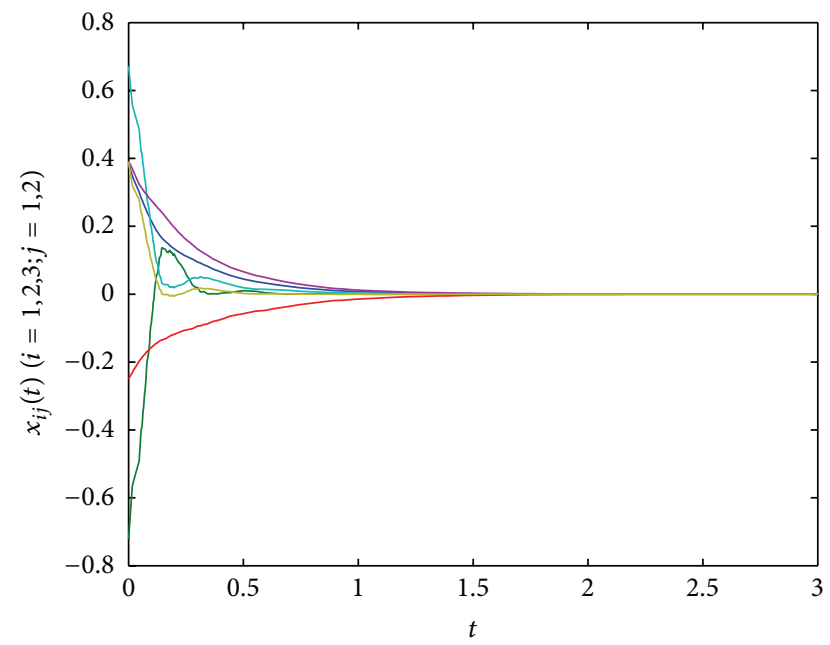

FIGURE 1: The position state curves of multiagent systems with $\alpha_{0}=$ $0.7, \mu=1$, and $\sigma=0$ (without failure).

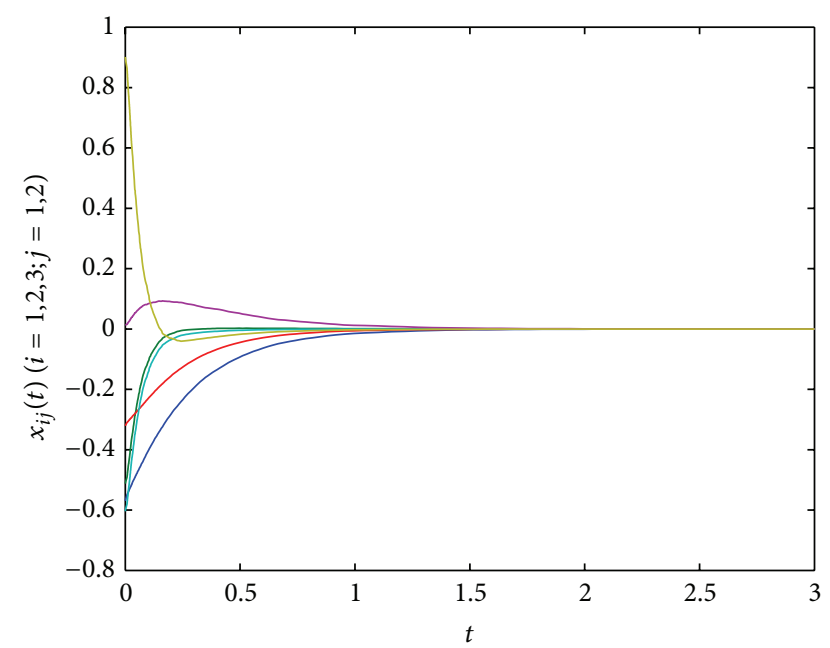

FIGURE 2: The position state curves of multiagent systems with $\alpha_{0}=$ $0.7, \mu=0.7$, and $\sigma=0.2$ (with partial failure).

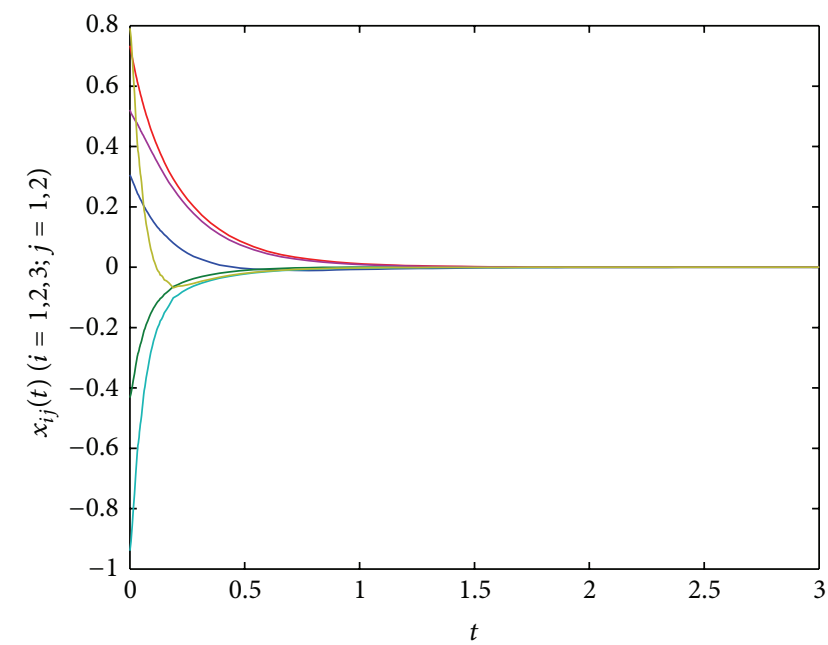

FIgURE 3: The position state curves of multiagent systems with $\alpha_{0}=$ $0.7, \mu=1.4$, and $\sigma=0.2$ (measurement distortion).

Theorem 12, the upper bound of the delay (sampling period) is obtained as $\tau=0.1063$ and the feedback gain is

$$
K=\left[\begin{array}{cc}
25.5233 & 14.9789 \\
-114.2535 & -172.8522
\end{array}\right] \text {. }
$$

The state responses are shown in Figure 3.

From the above three cases, when the actuator has fault, the upper bound of the delay (sampling period) is smaller than the other two cases. To illustrate the influence on the system by employing distribution information, for different $\alpha_{0}$, the upper bound of the delay (sampling period) and the corresponding feedback gain are listed in Tables 2 and 3 for without failure, partial failure, and measure distortion of the actuator.

\section{Conclusions}

The paper studies sampled-data consensus for nonlinear multiagent dynamical systems. A distributed linear reliable sampled-data consensus protocol is designed, where the 
actuator fault and random network-induced delay are considered. By using Lyapunov stability theory, some consensus conditions are derived in terms of linear matrix inequalities. In our future work, we will further consider the problems for randomly occurring nonlinearities and randomly occurring network topology.

\section{Conflict of Interests}

The author declares that there is no conflict of interests regarding the publication of this paper.

\section{Acknowledgments}

This work was jointly supported by National Natural Science Foundation of China under Grant nos. 61272034 and 11301226, Zhejiang Provincial Natural Science Foundation of China under Grant nos. LY13F030012 and LQ13A010017.

\section{References}

[1] R. W. Beard, T. W. McLain, M. A. Goodrich, and E. P. Anderson, "Coordinated target assignment and intercept for unmanned air vehicles," IEEE Transactions on Robotics and Automation, vol. 18, no. 6, pp. 911-922, 2002.

[2] J. A. Fax and R. M. Murray, "Information flow and cooperative control of vehicle formations," IEEE Transactions on Automatic Control, vol. 49, no. 9, pp. 1465-1476, 2004.

[3] W. Ren, R. W. Beard, and E. M. Atkins, "Information consensus in multivehicle cooperative control," IEEE Control Systems Magazine, vol. 27, no. 2, pp. 71-82, 2007.

[4] J. Cortés and F. Bullo, "Coordination and geometric optimization via distributed dynamical systems," SIAM Journal on Control and Optimization, vol. 44, no. 5, pp. 1543-1574, 2005.

[5] A. Jadbabaie, J. Lin, and A. S. Morse, "Coordination of groups of mobile autonomous agents using nearest neighbor rules," IEEE Transactions on Automatic Control, vol. 48, no. 6, pp. 988-1001, 2003.

[6] R. Olfati-Saber and R. M. Murray, "Consensus problems in networks of agents with switching topology and time-delays," IEEE Transactions on Automatic Control, vol. 49, no. 9, pp. 15201533, 2004.

[7] W. Ren and R. W. Beard, "Consensus seeking in multiagent systems under dynamically changing interaction topologies," IEEE Transactions on Automatic Control, vol. 50, no. 5, pp. 655661, 2005.

[8] W. Yu, G. Chen, and M. Cao, "Consensus in directed networks of agents with nonlinear dynamics," IEEE Transactions on Automatic Control, vol. 56, no. 6, pp. 1436-1441, 2011.

[9] G. Wen, Z. Duan, W. Yu, and G. Chen, "Consensus in multiagent systems with communication constraints," International Journal of Robust and Nonlinear Control, vol. 22, no. 2, pp. 170182, 2012.

[10] W. Yu, W. Ren, W. X. Zheng, G. Chen, and J. Lü, "Distributed control gains design for consensus in multi-agent systems with second-order nonlinear dynamics," Automatica, vol. 49, no. 7, pp. 2107-2115, 2013.

[11] G. Wen, G. Hu, W. Yu, J. Cao, and G. Chen, "Consensus tracking for higher-order multi-agent systems with switching directed topologies and occasionally missing control inputs," Systems \& Control Letters, vol. 62, no. 12, pp. 1151-1158, 2013.

[12] H. Zhao, J. H. Park, and Y. Zhang, "Couple-group consensus for second-order multi-agent systems with fixed and stochastic switching topologies," Applied Mathematics and Computation, vol. 232, pp. 595-605, 2014.

[13] T. H. Lee, J. H. Park, D. H. Ji, and H. Y. Jung, "Leader-following consensus problem of heterogeneous multi-agent systems with nonlinear dynamics using fuzzy disturbance observer," Complexity, vol. 19, no. 4, pp. 20-31, 2014.

[14] E. Fridman, U. Shaked, and V. Suplin, "Input/output delay approach to robust sampled-data $H_{\infty}$ control," Systems \& Control Letters, vol. 54, no. 3, pp. 271-282, 2005.

[15] E. Fridman, "A refined input delay approach to sampled-data control," Automatica, vol. 46, no. 2, pp. 421-427, 2010.

[16] Y. Cheng and D. Xie, "Distributed observer design for bounded tracking control of leader-follower multi-agent systems in a sampled-data setting," International Journal of Control, vol. 87, no. 1, pp. 41-51, 2014.

[17] M. J. Park, O. M. Kwon, J. H. Park, and S. M. a. Lee, "Randomly changing leader-following consensus control for Markovian switching multi-agent systems with interval timevarying delays," Nonlinear Analysis: Hybrid Systems, vol. 12, pp. 117-131, 2014.

[18] G. Guo, L. Ding, and Q.-L. Han, "A distributed event-triggered transmission strategy for sampled-data consensus of multiagent systems," Automatica, vol. 50, no. 5, pp. 1489-1496, 2014.

[19] T. Li and J. Zhang, "Sampled-data based average consensus control for networks of continuous-time integrator agents with measurement noises," in Proceedings of the 26th Chinese Control Conference (CCC '07), pp. 716-720, July 2007.

[20] H. Liu, G. Xie, and L. Wang, "Necessary and sufficient conditions for solving consensus problems of double-integrator dynamics via sampled control," International Journal of Robust and Nonlinear Control, vol. 20, no. 15, pp. 1706-1722, 2010.

[21] Y. Cao and W. Ren, "Multi-vehicle coordination for doubleintegrator dynamics under fixed undirected/directed interaction in a sampled-data setting," International Journal of Robust and Nonlinear Control, vol. 20, no. 9, pp. 987-1000, 2010.

[22] Y. Gao and L. Wang, "Sampled-data based consensus of continuous-time multi-agent systems with time-varying topology," IEEE Transactions on Automatic Control, vol. 56, no. 5, pp. 1226-1231, 2011.

[23] W. Yu, L. Zhou, X. Yu, J. Lu, and R. Lu, "Consensus in multiagent systems with second-order dynamics and sampled data," IEEE Transactions on Industrial Informatics, vol. 9, no. 4, pp. 2137-2146, 2013.

[24] W. Yu, W. X. Zheng, G. Chen, W. Ren, and J. Cao, "Second-order consensus in multi-agent dynamical systems with sampled position data," Automatica, vol. 47, no. 7, pp. 1496-1503, 2011.

[25] J. Zhang, X. Xu, L. Hong, and Y. Yan, "Consensus recovery of multi-agent systems subjected to failures," International Journal of Control, vol. 85, no. 3, pp. 280-286, 2012.

[26] J.-W. Wang and L.-L. Rong, "Edge-based-attack induced cascading failures on scale-free networks," Physica A: Statistical Mechanics and Its Applications, vol. 388, no. 8, pp. 1731-1737, 2009.

[27] Z. Wang, F. Yang, D. W. C. Ho, and X. Liu, "Robust $\mathrm{H}_{\infty}$ filtering for stochastic time-delay systems with missing measurements," IEEE Transactions on Signal Processing, vol. 54, no. 7, pp. 25792587, 2006. 
[28] Z. Wang, D. W. C. Ho, Y. Liu, and X. Liu, "Robust $H_{\infty}$ control for a class of nonlinear discrete time-delay stochastic systems with missing measurements," Automatica, vol. 45, no. 3, pp. 684-691, 2009.

[29] E. Tian, D. Yue, T. C. Yang, Z. Gu, and G. Lu, “TS fuzzy modelbased robust stabilization for networked control systems with probabilistic sensor and actuator failure," IEEE Transactions on Fuzzy Systems, vol. 19, no. 3, pp. 553-561, 2011.

[30] E. Tian, D. Yue, and C. Peng, "Brief Paper: reliable control for networked control systems with probabilistic sensors and actuators faults," IET Control Theory \& Applications, vol. 4, no. 8, pp. 1478-1488, 2010.

[31] E. Tian, D. Yue, and C. Peng, "Reliable control for networked control systems with probabilistic actuator fault and random delays," Journal of the Franklin Institute, vol.347, no. 10, pp. 19071926, 2010.

[32] D. Yue, E. Tian, Y. Zhang, and C. Peng, "Delay-distributiondependent robust stability of uncertain systems with timevarying delay," International Journal of Robust and Nonlinear Control, vol. 19, no. 4, pp. 377-393, 2009.

[33] D. Yue, E. Tian, and Y. Zhang, "A piecewise analysis method to stability analysis of linear continuous/discrete systems with time-varying delay," International Journal of Robust and Nonlinear Control, vol. 19, no. 13, pp. 1493-1518, 2009.

[34] Y. Liu, Z. Wang, J. Liang, and X. Liu, "Synchronization and state estimation for discrete-time complex networks with distributed delays," IEEE Transactions on Systems, Man, and Cybernetics B, vol. 38, no. 5, pp. 1314-1325, 2008.

[35] Y. Wang, Z. Wang, and J. Liang, "Global synchronization for delayed complex networks with randomly occurring nonlinearities and multiple stochastic disturbances," Journal of Physics A: Mathematical and Theoretical, vol. 42, no. 13, Article ID 135101, 2009.

[36] H. Li and D. Yue, "Synchronization of Markovian jumping stochastic complex networks with distributed time delays and probabilistic interval discrete time-varying delays," Journal of Physics A: Mathematical and Theoretical, vol. 43, no. 10, Article ID 105101, 2010. 


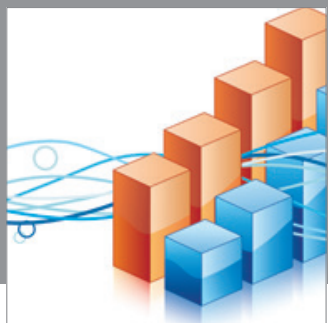

Advances in

Operations Research

mansans

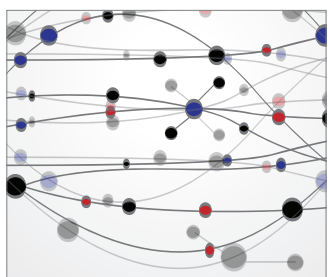

The Scientific World Journal
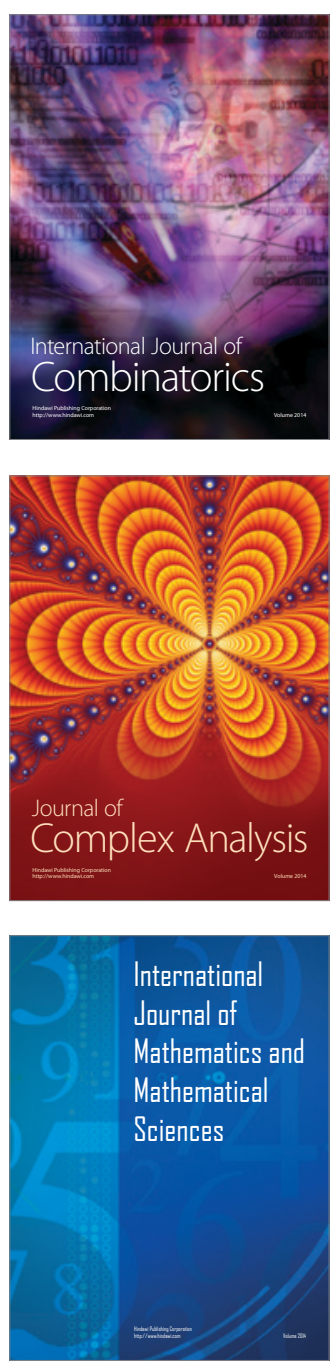
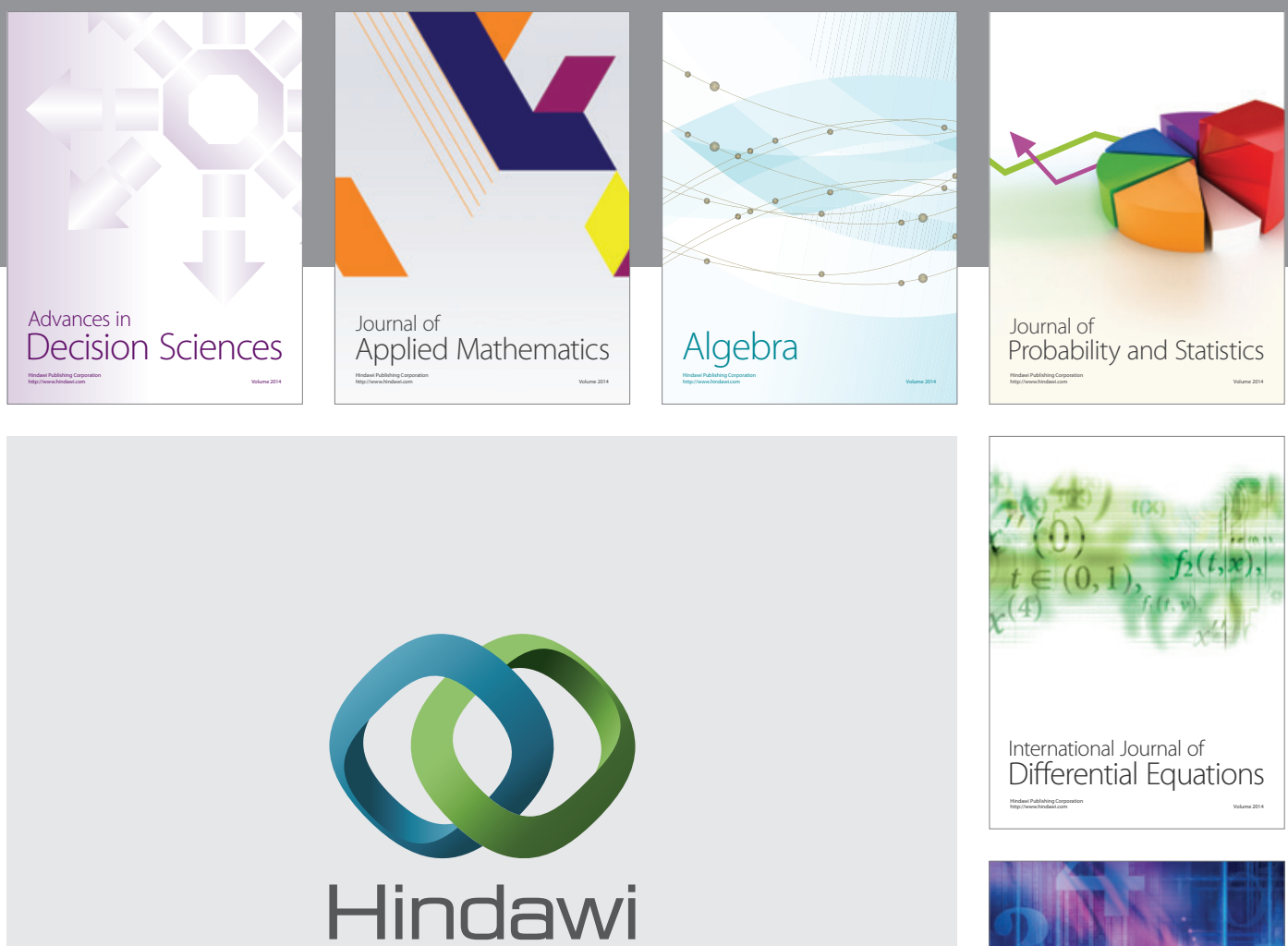

Submit your manuscripts at http://www.hindawi.com
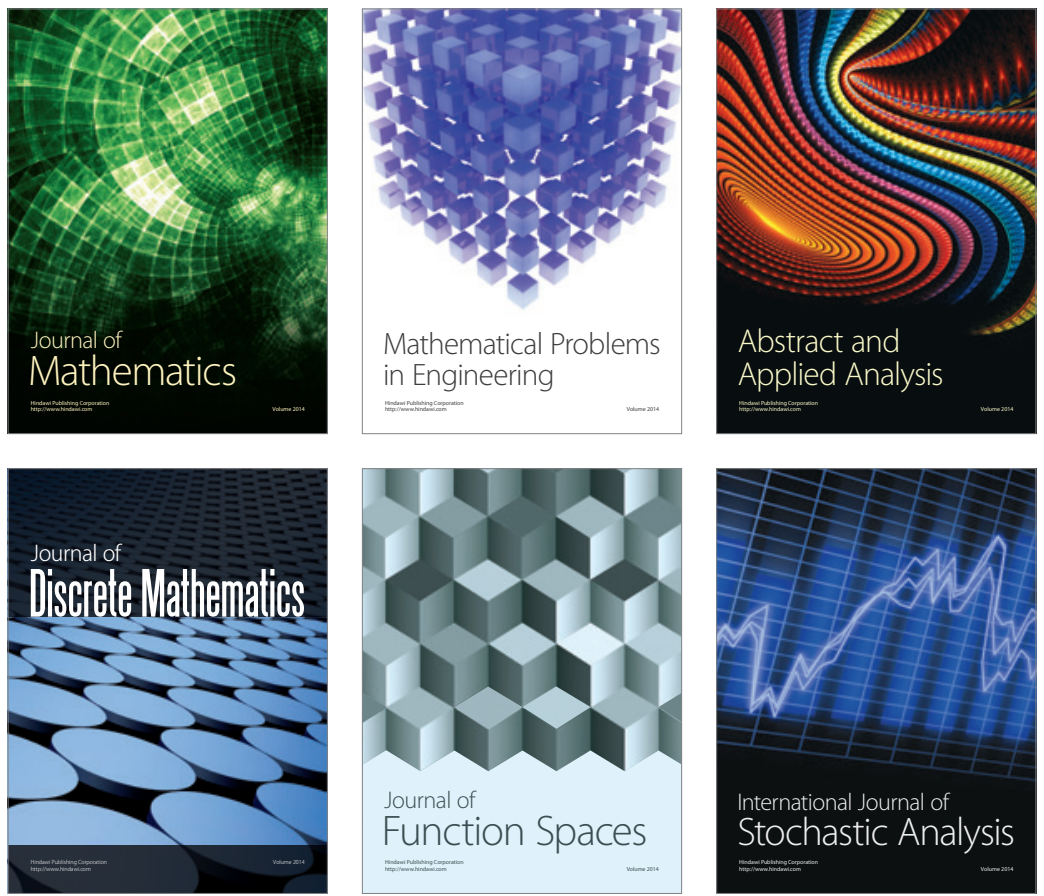

Journal of

Function Spaces

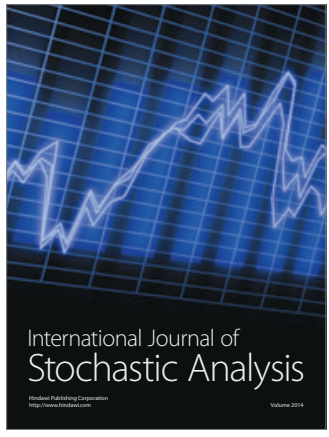

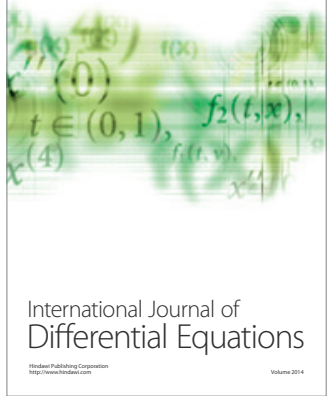
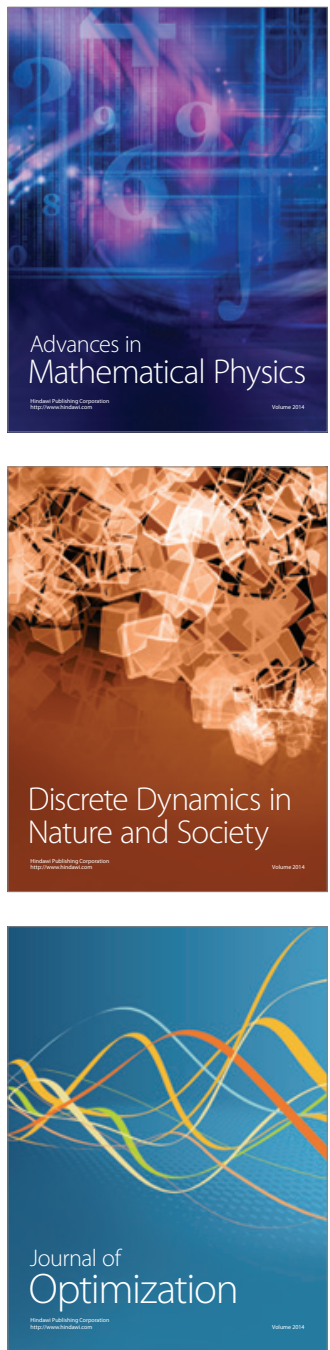